\title{
Cytology abnormal of cerebrospinal fluid in superficial siderosis of the central nervous system
}

\author{
Cui Wang, Jian-Xia Diao, Shu-Min Li \\ Department of Neurology, Dalian Municipal Central Hospital, Dalian 116033, Liaoning, China.
}

Correspondence to: Dr. Cui Wang, Department of Neurology, Dalian Municipal Central Hospital, Dalian 116033, Liaoning, China.

E-mail: wangc817@163.com

How to cite this article: Wang C, Diao JX, Li SM. Cytology abnormal of cerebrospinal fluid in superficial siderosis of the central nervous system. Neuroimmunol Neuroinflammation 2017;4:155-7.

\author{
Article history: \\ Received: 12-01-2017 \\ Accepted: 13-04-2017 \\ Published: 09-08-2017

\section{Key words:} \\ Superficial siderosis of the central \\ nervous system, \\ cytology of cerebrospinal fluid, \\ magnetic resonance imaging
}

\begin{abstract}
Superficial siderosis of the central nervous system (SSCNS) is usually caused by chronic subarachnoid hemorrhage which leads to the accumulation of hemosiderin in the subpial layers of the brain and the spinal cord. The exact clinical manifestations and T2-weighted magnetic resonance imaging (MRI) the patient presented here is diagnosed SSCNS mainly due to the cytology of cerebrospinal fluid (CCSF) and the superficial siderosis of T2-weighted MRI. CCSF can be a good complementary to the diagnosis of SSCNS.
\end{abstract}

\section{INTRODUCTION}

Superficial siderosis of the central nervous system (SSCNS) is a rare disorder that is resulted from recurrent and persistent hemorrhage which leads to the accumulation of hemosiderin in the surface of the brain and the spinal cord. These are typically clinical syndromes: sensorineural deafness, cerebellar ataxia, dementia and positive signs of pyramidal tract. Here, we describe one case of a 72-year-old male patient who was diagnosed with SSCNS using magnetic resonance imaging (MRI) and cytology of cerebrospinal.

\section{CASE REPORT}

The patient was 72-year-old and presented with a 3-month history of progressive sensorineural hearing loss, cerebellar ataxia, several absence seizures and dizziness and was admitted in our hospital. No relevant trauma or acute symptom onset was reported. He had no particular hobbies and there was no significant family history of neurological illness. 

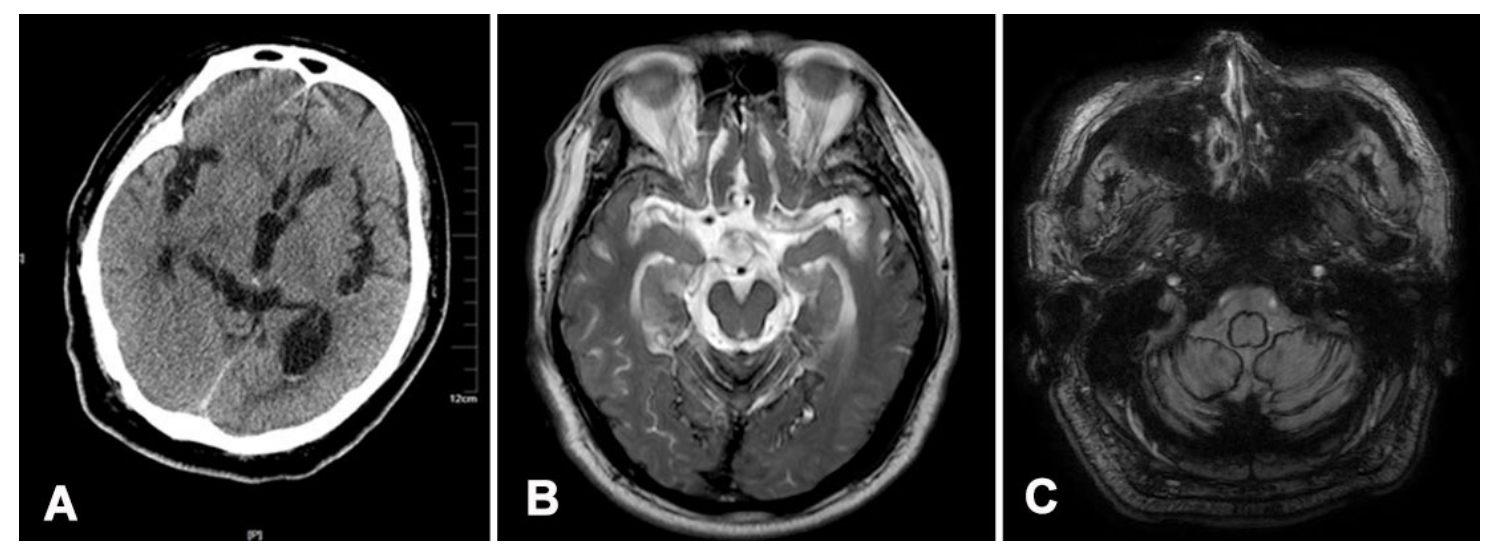

Figure 1: (A) Computed tomography scan showed that there was some blood in the posterior horn of the lateral ventricle; (B) T2-WI showing linear hypointensities along the surfaces of the brainstem and cerebellum; (C) susceptibility weighted imaging showing a dark rim around the cerebellar lobes and midbrain brain, corresponding to hemosiderin deposition

Abnormalities on neurological examination included decreased hearing in both ears, positive Babinski's sign on the both sides, and a wide-based gait. He was unable to maintain stable when asked to keep his eyes closed. Routine laboratory tests were normal. Computed tomography (CT) scan showed high density in the posterior horn of the lateral ventricle, and bilateral subarachnoid gap widened [Figure 1A]. No parenchymal hemorrhage was seen in the CT scan, T2-weighted and susceptibility weighted MR images (SWI) of the brain [Figure 1B and C] showed areas of linear hypointensity along the sylvian fissures, cortical sulci, surfaces of the brainstem and cerebellum. There was also evidence of significant cerebellar atrophy. Low signal could be seen in the posterior horn of the lateral ventricle form SWI [Figure 1C]. Extrallinear low signal on sylvian fissures and cerebral cortical sulci could be observed in SWI images. Lumbar puncture was performed, and it revealed the normal pressure of $140 \mathrm{mmH}_{2} \mathrm{O}$. The color of the cerebrospinal fluid (CSF) was pale. And CSF analysis showed 46,050

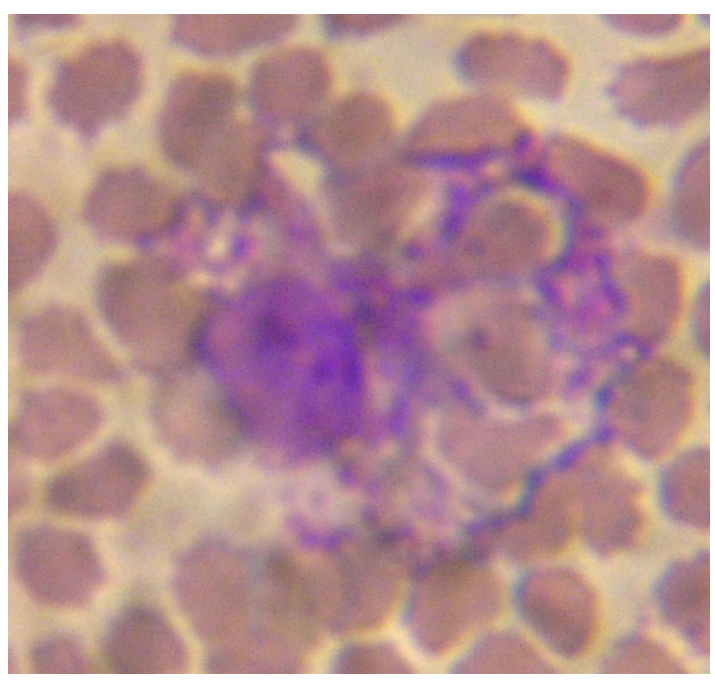

Figure 2: Cerebrospinal fluid cytology. A different number of red blood cells could be seen (MGG staining, $\times 1,000$ ) red blood cells (RBCs) $/ \mathrm{mm}^{3}$ and 28 white blood cells (WBCs) $/ \mathrm{mm}^{3}$. Sugar in CSF was normal. Chloride is $111 \mathrm{mmol} / \mathrm{L}$, protein was $1,324 \mathrm{mg} / \mathrm{L}$. Immunoglobulin was normal in CSF. The acid-fast stain and ink smears were negative. We gave our patient an additional CSF cytology (CCSF). CCSF revealed that the white blood cell counted $30 / \mathrm{mm}^{3}$, the red blood cell number counted 50,000 cells $/ \mathrm{mm}^{3}$. May-Grunwald-Giemsa staining was conducted and no abnormal shaped cells were seen. Lymphocytes were $54 \%$, monocytes were $14 \%$, neutrophile granulocytes were $27 \%$. We could see RBC phagocytes $1 \%$, hemosiderin phagocytes 4\% [Figure 2]. Electroencephalography showed the sharp waves in the focal right antero-mid temporal together with normal background. The patient was then diagnosed with SSCNS, subarachnoid hemorrhage and epilepsy. The reason of bleeding from subarachnoid hemorrhage was not detected after detailed examinations including digital subtraction angiography of brain and spinal cord.

\section{DISCUSSION}

SSCNS is an uncommon disorder. Until 2006 less than 300 cases of SSCNS have been reported..$^{[1,2]}$ Hemosiderin deposits in the subpial layers of the spinal cord and the brain. The pathogenesis of SSCNS was not cleanly known by us. So far the general thought is that excessive iron from recurrent subdural bleeding leads to the loss of neurons and myelin, resulting in the development of a neurological deficit. ${ }^{[3]}$ The most likely explanation for SSCNS is recurrent bleeding in the subarachnoid space. It may have lots of causes, including idiopathic (35\%), CNS tumor (15\%), head trauma $(13 \%)$, arteriovenous malformation $(9 \%)$. It is rarely seen in intradural neurosurgical operations, brachial plexus injury, nerve root avulsion, or other causes of subarachnoid hemorrhage (SAH). ${ }^{[4-6]}$ In our case, the patient refused to the whole spinal 
MRI. We were unable to identify the exact source of the bleeding, however, bleeding was present in the subarachnoid space.

T2-weighted MR imaging typically reports hypointensity along the surface of cerebellum and brainstem, corresponding to the deposition of hemosiderin. Cerebellar atrophy is also commonly associated with SSCNS. ${ }^{[7]}$ In our case, T2-weighted images showed a rim of hypointensity around the cerebellum, brainstem, and Sylvian fissure, which helped us to diagnose this patient with SSCNS. In recent years, RBCs have been observed in CSF studies with SSCNS. The presence of RBCs in the CSF is the defining feature of SSCNS. A non-traumatic lumbar puncture was performed in our patient, the color was pale red. CSF was full of characteristics following analysis and cytology. It showed a large number of RBCs and obvious neutrophilic response. There were erythrocyte phagocytes and hemosiderin phagocytic cells measure in the CCSF. Seven days later, we were able to see bilirubin phagocytes. All the above types of phagocytic cells suggested that the cerebral hemorrhage had not stopped or might be a sign of rebleeding ${ }^{[8]}$ This kind of performance was consistent with its pathogenesis and pathophysiology. From what has been discussed above, CCSF can prompt the pathogenesis of the SSCNS, it was identified a beneficial supplement to CSF analysis. We come to a conclusion that CCSF can provide one of the objective bases of clinical diagnosis of SSCNS.

At present, we have no effective treatment for SSCNS. ${ }^{[9]}$ Surgical treatment can be used to remove the potential source of recurrent $\mathrm{SAH}$ in order to arrest the clinical deterioration, but a number of patients has been reported to have experienced aggravated symptoms after surgery. ${ }^{[10]}$ Iron chelating drugs were identified ineffective because of the bloodbrain barrier (BBB). Recently, Schirinzi et al. ${ }^{[11]}$ used soluble deferiprone that could cross BBB. He found that soluble deferiprone can improve ataxia. The CSF-ferritin concentration could be slightly reduced. However, the neuroimaging remained unchanged. After administering our patient with carbamazepine $100 \mathrm{mg}$ three times a day, epilepsy symptoms disappeared. We could not find the cause of bleeding, no surgical intervention was performed. Now (1.5 years from the last discharge), he is still alive and sensorineural hearing loss. His cognitive function has badly declined and reports of always being in bed and needs the assistance of family members. He also reports of incontinence, but no reports of seizures. CCSF findings revealed the etiology and pathology mechanism of SSCNS. T2-weighted MRI and SWI showed that reliable changes of the head imaging. Combined with the patient's clinical manifestations, the diagnosis of SSCNS was obtained. T2-weighted MRI used together with the CCSF is strongly recommended in differential diagnosis of SSCNS.

\section{DECLARATIONS}

\section{Authors' contributions}

Conception, diagnosis and design: C. Wang

Manuscript preparation: J.X. Diao

Pathology diagnosis: S.M. Li

Manuscript revision: C. Wang

Final approval of manuscript: C. Wang

\section{Financial support and sponsorship} None.

\section{Conflicts of interest}

There are no conflicts of interest.

\section{Patient consent \\ Patient consent was obtained from the patient.}

\section{Ethics approval}

There is no ethics issue in this paper.

\section{REFERENCES}

1. Fearnley JM, Stevens JM, Rudge P. Superficial siderosis of the central nervous system. Brain 1995;118:1051-66.

2. Kumar N. Neuroimaging in superficial siderosis: an in-depth look. AJNR Am J Neuroradiol 2010;31:5-14.

3. Ting SK, Prakash KM. A rare cause of cerebellar ataxia syndrome: superficial siderosis of central nervous system. Acta Neurol Taiwan 2011;20:257-61.

4. Zhao H, Wang J, Lu Z, Wu Q, Lv H, Liu H, Gong X. Superficial siderosis of the central nervous system induced by a single-episode of traumatic subarachnoid hemorrhage: a study using MRIenhanced Gradient Echo T2 star-weighted angiography. PLoS One 2015;10:e0116632.

5. Levy M, Turtzo C, Llinas RH. Superficial siderosis: a case report and review of the literature. Nat Clin Pract Neurol 2007;3:54-8.

6. Koeppen AH, Michael SC, Li D, Chen Z, Cusack MJ, Gibson WM, Petrocine SV, Qian J. The pathology of superficial siderosis of the central nervous system. Acta Neuropathol 2008;116:371-82.

7. Yamawaki T, Sakurai K. Diagnosis and treatment of superficial siderosis. Brain Nerve 2013;65:843-55. (in Japanese)

8. Bigner SH. Cerebrospinal fluid (CSF) cytology: current status and diagnostic applications. J Neuropathol Exp Neurol 1992;51:235-45.

9. Kumar N. Superficial siderosis: associations and therapeutic implications. Arch Neurol 2007;64:491-6.

10. Payer M, Sottas C, Bonvin C. Superficial siderosis of the central nervous system: secondary progression despite successful surgical treatment, mimicking amyotrophic lateral sclerosis. Case report and review. Acta Neurochir (Wien) 2010;152:1411-6.

11. Schirinzi T, Sancesario G, Anemona L, Pisani A, Sancesario G. CSF biomarkers in superficial siderosis: a new tool for diagnosis and evaluation of therapeutic efficacy of deferiprone -- a case report. Neurol Sci 2014;35:1151-2. 